\title{
Análise do ecoturismo como fonte de pesquisa
}

\author{
Brenda Oliveira Nascimento ${ }^{1}$ \\ Roberto Gondim Pires ${ }^{2}$ \\ Robelius De-Bortoli ${ }^{1}$ \\ ${ }^{1}$ Universidade Federal de Sergipe, Laboratório de Pesquisa do Desenvolvimento Cognitivo Humano. \\ Sergipe, Brasil. \\ 2 Universidade Estadual do Sudoeste da Bahia, Campus de Jequié, Departamento de Saúde. Bahia, \\ Brasil.
}

ORIGINAL

\begin{abstract}
Resumo
Objetivo. Identificar quantitativamente a produção acerca do Ecoturismo, bem como os responsáveis por esses materiais, proporcionando aos pesquisadores uma fonte acessível de informações sobre as publicações e sobre os envolvidos nos materiais relativos à temática.

Método. Trata-se de uma pesquisa quantitativa e qualitativa, e utiliza como método a Bibliometria. O material coletado para este estudo foi capturado por meio da plataforma de dados do 'Google Acadêmico' e foi dividida em 03 etapas, sendo elas, a coleta do material, a triagem do material escolhido, e a tabulação dos dados e elaboração dos gráficos com utilização do software 'Microsoft Excel'.

Resultados. Os dados encontrados resultaram em 25 plataformas diferentes, apresentando um total de 40 artigos sobre a temática investigada. As análises dos dados remeteram à possível relação entre indivíduos responsáveis pelas plataformas com os autores dos artigos, isto revela uma possível fragilidade da integridade das avaliações e publicações de materiais científicos, mostrando que há possibilidade de um estreitamento das relações entre periódicos, autores e avaliadores, podendo provocar com isso um ciclo vicioso, ou endógeno.

Conclusões. Esse trabalho demostra uma preocupação frente à propagação de ideias que eleve esse tema, mas que por outro lado possa causar a estagnação do mesmo. O Ecoturismo é um tema que está em ascensão e que aguça o interesse de novos pesquisadores. Considerando a possibilidade de endogenia, essa prática resulta no enfraquecimento e essa fragilidade pode promover a sua estagnação ao invés de seu progresso conceitual.
\end{abstract}

Palavras-chave

Bibliometria. Ética em pesquisa. Pesquisa.

\section{Ecotourism's analysis as source of research}

\begin{abstract}
Objective. Quantitatively identify the production about ecoturism as well as those responsible for these materials, providing researchers with an accessible source of information on publications and those related to theme materials.

Method. It is a quantitative and qualitative research, and uses Bibliometric as method. The material collected for this study was captured through the "Google Scholar" data platform and was divided into three stages: the collection of the material, the sorting of the chosen material and the tabulation of the data and the drawing of the graphs with the use of Microsoft software Excel.

Results. The data found resulted in 25 different platforms, presenting a total of 40 articles on the subject searched. The analysis of the data referred to the possible relationship between individuals responsible for the platforms with the authors of the articles, which reveals a possible fragility of the integrity of the evaluations and publications of scientific materials, showing the possibility of a closer relationship between magazines, authors and Evaluators, and can thus cause a vicious cycle, or endogenous.

Conclusions. This work demonstrates a concern in the face of the propagation of ideas that elevate this theme, but that on the other hand may cause its stagnation. Ecotourism is a theme that is on the rise and that sharpens the interest of new researchers. Considering the possibility of endogeny, this practice results in weakening and this fragility may promote its stagnation instead of its conceptual progress.
\end{abstract}

Keywords

Bibliometric. Ethics. Research. 


\section{Introdução}

Vasconcelos (2003) apontava que o ecoturismo era um movimento recente e que sua prática auxilia no desenvolvimento dos programas de educação ambiental, visando a conscientização dos visitantes. Ao fomentar essa discussão, o autor retrata os impactos ambientais negativos causados pelas atividades turísticas no meio natural descaracterizando a naturalidade de ambientes. Assim, o Ecoturismo surge como uma proposta para beneficiar tanto a natureza quanto a população. Sua prática, além de ser direcionada como um alerta para conservação ambiental, tem preocupação com o bem-estar proporcionado pelo contato com a natureza (CARVALHO, 2016).

O documento "Diretrizes para uma Política Nacional de Ecoturismo", publicado pelo Ministério do Meio Ambiente [MMA] em conjunto com a Empresa Brasileira de Turismo [EMBRATUR], estabelece o conceito do ecoturismo como iniciativa voltada para a conservação ambiental e preocupação com as comunidades locais, citando o Ecoturismo como

"[...] um segmento da atividade turística que utiliza, de forma sustentável, o patrimônio natural e cultural, incentiva sua conservação e busca a formação de uma consciência ambientalista através da interpretação do ambiente, promovendo o bem-estar das populações envolvidas" (MMA, 1994, p.19).

O Ecoturismo caracteriza-se como turismo alternativo e ao mesmo tempo distinto do turismo convencional, em que se destaca no cenário de conservação ambiental pois valoriza uma relação mais intima com o meio ambiente e com as comunidades locais. Castro, Noronha e Medeiros (2016) afirmam que o ecoturismo mostrase como um aliado na gestão ambiental de áreas de relevância, ao mesmo tempo em que fortalece a apropriação das áreas naturais, dinamiza as economias locais e incrementa os recursos financeiros para a manutenção destas áreas.

Vale ressaltar que o Ecoturismo surge não somente com o intuito de preservação ambiental e/ou conservação ambiental, mas também para auxiliar as comunidades dessas regionalidades a obterem renda para continuarem desenvolvendo suas atividades. Isso deveria ocorrer de modo sustentável, proporcionando um crescimento econômico planejado e adequado, reduzindo os impactos ambientais e sem prejuízos para a comunidade receptora. Essa valorização do Ecoturismo deveu-se à criação das Unidades de Conservação [UCs].

Segundo Kundlatsch, Vieira e Moreira (2016), essa valorização se deve aos seguintes motivos: o desenvolvimento de atividades turísticas (lazer, descanso, relaxamento e aventura, que fogem da correria do cotidiano urbano) e das educativas (como práticas de percepção e interpretação ambiental). Elas proporcionariam o contato direto com a natureza, por trazerem a reflexão sobre a relação sociedade e natureza contemporânea, a complexa problemática ambiental dessa relação e a necessidade da conservação e preservação.

Observando-se essas peculiaridades do Ecoturismo, é notória sua preocupação com ações de preservação ambiental, mas não fugindo da característica de visitação de ambientes naturais que geram conhecimento, renda a comunidade local, bem-estar e qualidade de vida por meio dos locais explorados. Essa relevância socioambiental e econômica que é gerada por meio do Ecoturismo tende a despertar o interesse de pesquisadores sobre essa modalidade.

Dessa forma, este estudo se enquadra no tipo de pesquisa que visa investigar as perspectivas em que o Ecoturismo está imerso, objetivando assim, identificar quantitativamente a produção acerca dessa temática, bem como os responsáveis por estes materiais. Para obtenção desse resultado, busca-se verificar se há endogenia a partir do material publicado e da análise dos periódicos envolvidos.

Ao tentar elucidar tais perspectivas, faz-se necessário identificar quem são os autores e quais são as revistas que se debruçam sobre a temática Ecoturismo. Desta forma, o objetivo deste estudo é proporcionar aos pesquisadores uma fonte acessível de informações sobre as publicações e sobre os envolvidos nos materiais relativos ao Ecoturismo. 


\section{Metodologia}

$\mathrm{O}$ estudo optou por fazer uso da pesquisa quantitativa e qualitativa. $\mathrm{Na}$ abordagem qualitativa procurou-se aprofundar na compreensão dos fenômenos que estuda, enquanto que na abordagem quantitativa, preocupouse em medir (quantidade, frequência e intensidade) e analisar as relações causais entre as variáveis (TERENCE; ESCRIVÃO FILHO, 2006).

O método estabelecido consistiu na aplicação de técnicas estatísticas para a contagem e estabelecimento de padrões de regularidade em itens informacionais de edições e de autores que publicam em periódicos (ARAÚJO, 2009). Dessa forma, foi utilizado a Bibliometria em que fomenta a discussão sobre a construção do conhecimento da área, complementando as análises epistemológicas e metodológicas da produção científica (SACARDO; HAYASHI, 2010).

O material coletado para este estudo foi capturado por meio da plataforma de dados do 'Google Acadêmico'. Para realizar a captura do material a ser estudado e analisado, foram realizados os seguintes procedimentos: dentro da plataforma foi digitada a nomenclatura 'Ecoturismo', em seguida, utilizando as ferramentas para maximizar a busca, foi desativada a ferramenta 'incluir patentes' e também 'incluir citações', pesquisando por páginas em português, classificando por datas, mas sem estipular uma data inicial. Esse procedimento foi realizado para publicações até o dia 30 de setembro de 2016 .

O recorte adotado para delinear o campo que foi verificado nesta pesquisa foi trabalhar exclusivamente com textos veiculados em revistas, descartando assim, a possibilidade de manusear livros, teses, dissertações e anais de congresso. O volume da produção e o acesso a esses materiais tornam a pesquisa inviável, pois algumas teses e dissertações não são disponibilizadas online dificultando a captação das informações. No intuito de responder aos objetivos desse trabalho, optou-se, portanto, em analisar artigos de revistas eletrônicas, o que tornou a pesquisa viável.

Para realizar a identificação dos responsáveis pela revista, bem como os integrantes que fazem com que a mesma mantenha a análise do material encaminhado para publicação, fez-se necessário identificar quais os indivíduos que estão estudando e possibilitando a viabilidade do material aqui em discussão. Para obtenção de resultados eficazes para tal problemática, os integrantes foram divididos em dois grupos: Grupo A que foram alocados os: 'Editor(es)-Chefe', 'Editor Adjunto', 'Editor Executivo', 'Editor Responsável', 'Secretaria Executiva', 'Editores de Seção', 'Editores de Texto', 'Editor Cientifico', 'Editor Gerente', 'Assessores do Editor Científico', 'Editor Assistente', 'Editor de Artigos e Iniciação Científica', 'Editores Dossiê Literatura e Resistência', 'Editores Associados'; e Grupo B que foram alocados os 'Comitê Cientifico de Avaliadores', 'Comitê Editorial', 'Conselho Cientifico', 'Comitê de Assessoramento Científico', 'Corpo Editorial', 'Revisores Ad Hoc', 'Comitê de Ética', 'Conselho Editorial', 'Pareceristas', 'Conselho Editorial Internacional'.

A coleta do material foi dividida em 03 etapas. Na primeira etapa, foi realizada a coleta do material no banco de dados do 'Google Acadêmico' por meio da palavra Ecoturismo. Na segunda etapa, foi realizada a triagem do material escolhido de acordo com o que foi citado acima na metodologia. Na terceira etapa foram tabulados os dados e elaborados os gráficos com utilização do software Microsoft Excel.

Para o desenvolvimento da pesquisa, atentou-se para que fossem estabelecidos cuidados éticos referente a este trabalho, sendo eles a não exposição das respectivas revistas analisadas, a não exposição dos membros destas revistas, assim como também não revelar o nome dos autores que escrevem sobre a temática aqui pesquisada. Esses dados ficarão acessíveis somente aos pesquisadores e depois de 5 anos serão inutilizados.

\section{Resultados}

De acordo com a primeira etapa desse estudo, desconsiderando patentes, citações e pesquisando páginas em português, retornaram 94 ocorrências ao digitar a palavra 'Ecoturismo' na plataforma de busca do 'Google Acadêmico'. Na segunda etapa realizada, das 94 ocorrências encontradas na pesquisa, 54 estão localizadas em plataformas diferentes que abordam a temática pesquisada. Dentre estas, 25 são periódicos nos quais disponibilizam os artigos na íntegra e de forma digitalizada. Por motivos explícitos na metodologia deste 
trabalho, foram utilizadas apenas estas. Nas 25 revistas selecionadas a partir dos critérios pré-estabelecidos, obteve-se 40 artigos sobre a temática investigada, o Ecoturismo.

Das 25 revistas que fizeram parte desse trabalho bibliométrico, foram coletados dados referentes a equipe editorial de cada uma delas. Os dados extraídos foram os nomes dos representantes que são responsáveis pelas revistas e pela avaliação do material para publicação. Por existir uma variação e quantidade significativa de nomes designados aos representantes, os mesmos foram divididos em Grupo A e Grupo B, determinados na metodologia.

$\mathrm{Na}$ terceira e última etapa foi realizada a tabulação dos dados. Após a seleção do material, foram encontradas no Grupo A 71 pessoas responsáveis por ele, obtendo assim, 9\% da população total encontrada destinada a esse grupo. No Grupo B foram encontradas 925 pessoas responsáveis, distribuídas pelas nomenclaturas citadas de acordo com a metodologia, correspondendo assim, a $91 \%$ do total da população. Os dados podem ser mais bem visualizados na figura 1.

Figura 1. Relação quantitativa entre os membros do Grupo A e o Grupo B.

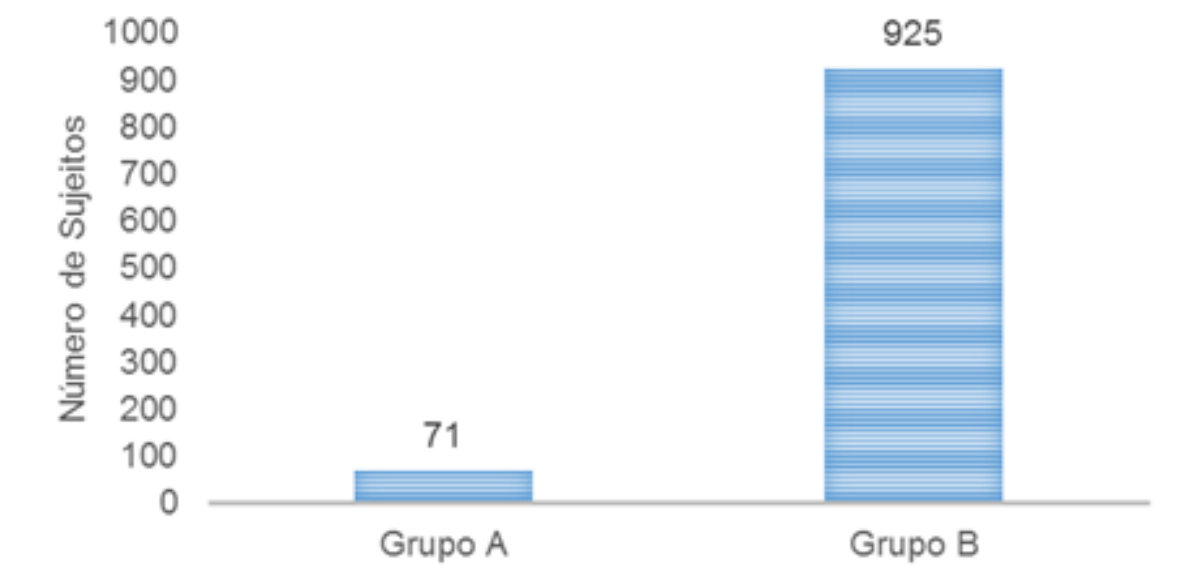

Fonte: Elaborado pelo próprio autor

No Grupo A, dos 71 sujeitos desse grupo, há uma repetição equivalente a 4 membros que aparecem em mais de uma revista entre as nomenclaturas referente a esse grupo. No Grupo B, dos 925 sujeitos que compõem esse grupo, 141 membros se repetem mais de uma vez, podendo ser mais bem visualizado a partir da figura 2.

Figura 2. Frequência dos sujeitos do Grupo A e do Grupo B em mais de uma revista.

\section{口GRUPO A IGRUPO B}

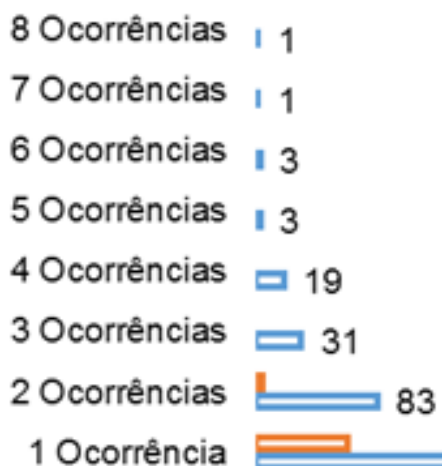

\section{Quantitativo de Sujeitos}


Também foi analisada a repetição entre os grupos, a fim de verificar se os sujeitos do Grupo A se repetem no Grupo B. Ao realizar o cruzamento dos dados com os nomes dos sujeitos dos dois grupos foi possível encontrar repetição desses representantes, como mostra a figura 3. Identificou-se quantitativamente que 10 sujeitos se repetem entre o Grupo A e o Grupo B, e 1 dos sujeitos têm seu nome repetido entre os dois grupos oito vezes. Sendo assim, foi detectada uma repetição considerável entre esses sujeitos e os grupos nos quais estão inseridos.

Figura 3. Quantitativo da repetição de sujeitos do Grupo A e do Grupo B.

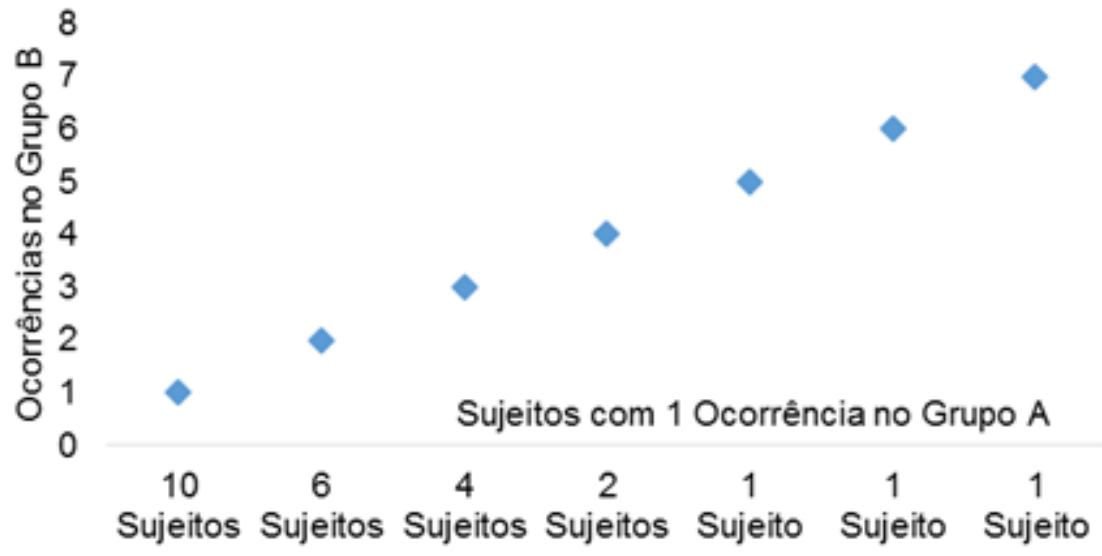

Fonte: Elaborado pelo próprio autor.

Para atender aos objetivos e para complementar a análise dos resultados, esse trabalho atentou-se em investigar a relação entre os sujeitos do Grupo A e do Grupo B sobre a temática pesquisada, o Ecoturismo. Desse modo, a análise dos dados buscou identificar se há alguma relação entre os autores dos artigos encontrados com os sujeitos do Grupo A e consequentemente o mesmo procedimento foi realizado com o Grupo B.

Em relação ao Grupo A, apenas 1 autor de um dos artigos foi identificado como um dos sujeitos presente no Grupo A. Já em relação ao Grupo B, é possível visualizar que possui um número maior de repetições entre esses sujeitos. Identificou-se que um autor aparece 9 vezes entre o quantitativo de sujeitos presentes no Grupo B. Esses dados podem ser melhor visualizados de acordo com sua apresentação no gráfico 4.

Figura 4. Quantitativo de repetições de autores e o Grupo A e o Grupo B.

\section{口Grupo B aGrupo A}

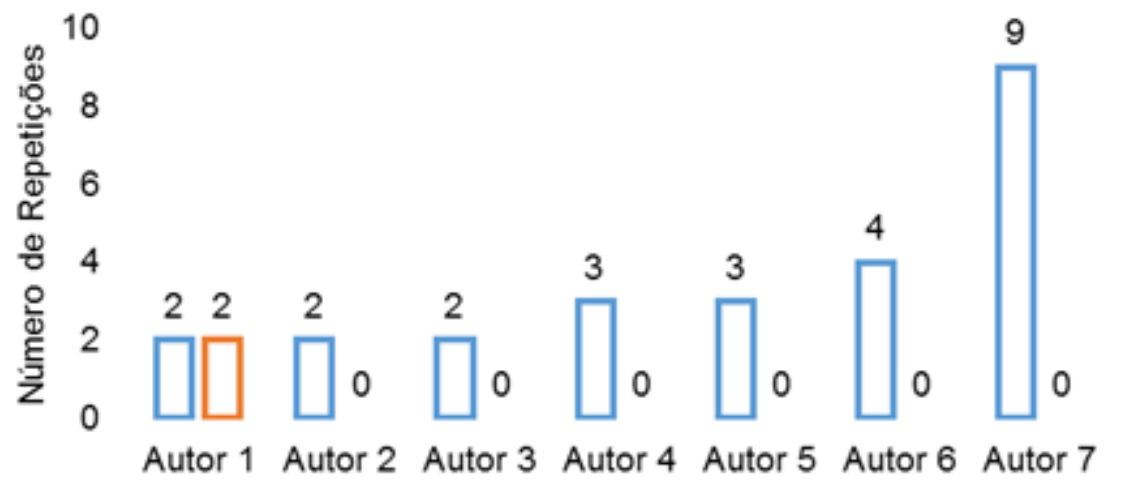

Fonte: Elaborado pelo próprio autor. 


\section{Discussão}

A seguir são interpretados os dados da base analisada, à partir das estratégias elencadas na metodologia utilizada neste estudo. Faz-se aqui o esclarecimento do que foi abarcado durante as análises, evidenciando assim os achados.

De acordo com as análises efetuadas, encontrou-se dados referentes ao quantitativo de representantes no Grupo A menor do que no Grupo B. Isso é fundamentado pelo que traz os Critérios, Política e Procedimentos para a admissão e a permanência de periódicos científicos na Coleção SciELO no Brasil (SCIELO, 2014). Nele são tratados pontos específicos para a solidificação de um periódico e aborda que todos os periódicos devem ter um ou mais editores-chefes definidos, com afiliação nacional ou estrangeira, sendo assim responsáveis pela gestão editorial e pelo desempenho final dos periódicos, tendo os editores como função principal referente ao desenvolvimento da política editorial e avaliação de manuscritos. Já em relação ao Grupo B, é notória a necessidade de um maior quantitativo de pessoas responsáveis por analisar e avaliar o material enviado por autores, ou seja, um leque de especialistas para avaliar os manuscritos, como também os próprios periódicos.

Dessa forma ao se pensar na estruturação de um periódico em que abarca os membros que estão representados pelo Grupo A e pelo Grupo B, pensa-se na visibilidade que o mesmo venha a obter. Com isso, Oliveira, Cendón e Cirino (2017), abordam que o periódico científico é um instrumento de disseminação da produção científica em área específica do conhecimento. Eles ressaltam que tão importante quanto disponibilizar os periódicos é estruturar as publicações para que possam ter condições básicas para obterem visibilidade nacional e internacional.

Foi publicado no Fórum Internacional de Turismo do Iguassu um estudo de Paula e Albach (2015) que analisou uma base de dados que, ao cruzar esses dados, não encontrou nenhuma repetição entre os autores. Contrariando esse dado, neste estudo são encontrados autores que são membros do Grupo A e do Grupo B, sendo que no Grupo B esse número foi mais elevado.

No $4^{\circ}$ Fórum Internacional Ecoinovar Santa Maria, RS, Kempat, Kreski, Freitas e Santos (2015) publicaram um estudo dedicado a realizar uma análise que indica que ambos os gêneros publicam sobre a temática e que a mesma vem crescendo, mas não aponta a repetição dos mesmos entre as publicações. De maneira contraditória, neste trabalho os dados indicam a repetição entre os membros dos dois grupos analisados, fazendo uma comparação entre os autores e os membros dos dois grupos investigados.

Nesta pesquisa foi encontrado repetição entre os membros do Grupo A e também do grupo B com os autores dos artigos. Costa e Guimarães (2010) em seu estudo tratam sobre a qualidade de periódicos científicos e determinam que podem ser avaliados com foco no processo relacionados com a gestão editorial ou no resultado. Torna-se relevante saber o nível de impacto exercido pelos artigos publicados, se contribuem para o fortalecimento de determinada área da comunidade científica, obtendo assim um maior nível de impacto. Seguindo essa linha de pensamento, quanto mais os artigos são citados pelos autores, maior seu nível de impacto.

Outro ponto que chama bastante atenção nessas análises é sobre a presença de autores que aparecem no Grupo A e no Grupo B. Apenas 1 autor foi identificado como membro do Grupo A. Já no Grupo B, esse número foi bem maior, pelo menos um autor aparece 9 vezes entre os membros do Grupo B. Nesse contexto, tal fator pode se apresentar como prejudicial a qualidade, em que pode se revelar como uma possível endogenia. Valério (1994), em seu livro Espelho da ciência [...], caracteriza endogenia tanto pela presença na revista de autores que pertencem a instituição editora, quanto pela realização de avaliações feitas por assessores científicos dessa mesma instituição. Sales (2013) aborda endogenia como a concentração institucional ou geográfica dos revisores e dos autores, observada pela afiliação declarada do conselho editorial com membros oriundos de uma mesma instituição, cidade ou região.

Esses conceitos de endogenia que foram apresentados estão muito próximos, e revelam a fragilidade da integridade das avaliações e publicações de materiais científicos, mostrando que há possibilidade de um estreitamento das relações entre periódicos, autores e avaliadores, podendo provocar com isso um ciclo vicioso, ou endógeno.

Valério (1994) já ressaltava sobre quão prejudicial isso se torna, apontando que essa condição não traz benefícios para a ciência, pois tal prática não viabiliza a renovação de ideias. Pelo contrário, isso presume a estagnação e propagação sempre das mesmas ideias, dos mesmos grupos e das mesmas pessoas responsáveis. Este pensamento também é corroborado por Braga e Venturini (2013), que ainda acrescentam 
que a literatura não é otimista visto as consequências provocadas pela endogenia e apontam consequências negativas referente a produtividade acadêmica como também para a qualidade do conhecimento.

Um aspecto que é importante e que é objeto deste estudo é a tentativa de esclarecer, a partir da análise bibliométrica, se é possível os membros do Grupo A estarem também presentes no Grupo B e vice-versa. Mas isso não quer dizer que estejam no mesmo periódico, principalmente devido às funções/cargos exercidos pelos membros tanto do Grupo A, quanto do Grupo B, o que não foi objeto de investigação deste trabalho podendo ser aqui uma limitação do estudo.

\section{Considerações finais}

Tendo como objetivo investigar o campo do Ecoturismo, bem como os envolvidos nessa temática, esse trabalho possibilitou uma análise do quadro dos responsáveis pelos respectivos periódicos aos quais são enviados e publicados os trabalhos, como também as pessoas que escrevem para tornar a temática acessível aos futuros pesquisadores.

Os dados aqui obtidos pelas análises bibliométricas possibilitaram uma discussão relevante sobre aspectos endógenos, tanto sobre a composição dos periódicos, quanto aos autores que se debruçam sobre a temática. Portanto, avalia-se uma endogenia que transcende a visão apenas institucional. A partir desse trabalho, visualiza-se que essa concentração vai além dos interesses institucionais, de acordo com a breve análise feita dos dados encontrados.

Esse trabalho demostra uma preocupação frente à propagação de ideias que eleve esse tema, mas que por outro lado possa causar a estagnação do mesmo. O Ecoturismo é um tema que está em ascensão e que aguça o interesse de novos pesquisadores. Considerando a possibilidade de endogenia, essa prática resulta no enfraquecimento e essa fragilidade pode promover a sua estagnação ao invés de seu progresso conceitual.

Uma limitação desse estudo, mas que pode ser aprofundado por outros pesquisadores, trata-se de identificar a influência direta ou indireta que os membros dos Grupos A e B podem exercer um sobre o outro, ou até mesmo referente aos próprios autores, indicando efetivamente a que tipo de afiliação se faz presente. Este trabalho oferece subsídios para realizar mais investigações aprofundadas sobre ações que poderiam ser consideradas endógenas.

\section{Referências}

ARAÚJO, C. A. A. Correntes teóricas da ciência da informação. Ciência da Informação, Brasília, DF, v. 38, n. 3, p.192-204, set./dez., 2009.

BRAGA, M. M. S.; VENTURINI, A. E. J. F. Endogenia acadêmica em um programa de pós-graduação em direito. Direito, educação, ensino e metodologia jurídicos. p. 91 - 108. Florianópolis: FUNJAB, 2013. Disponível em: http://www.publicadireito.com.br/artigos/?cod=30f0641c041f03d9

CARVALHO, T. L. G. O turismo no Morro da Babilônia: do reflorestamento ao Ecoturismo. Revista Brasileira de Ecoturismo, São Paulo, v.9, n.1, fev/abr, pp.11-28, 2016.

CASTRO, L. L. C.; NORONHA, G. S.; MEDEIROS, M. A. A. Ecoturismo como alternativa de Desenvolvimento Socioeconômico na llha do Cajual, Alcântara (MA). Revista Brasileira de Ecoturismo, São Paulo, v.9, n.3, ago/out, pp.418-432, 2016.

COSTA, S. M. DE S.; GUIMARÃES, L. V. DE S. Qualidade de periódicos científicos eletrônicos brasileiros que utilizam o sistema eletrônico de editoração de revistas (SEER). Informação \& informação. v. 15, n. esp., p. 75 - 93, Londrina, 2010.

KEMPAT, C. B.; KRESKI, A. T.; FREITAS, M. L.; SANTOS, W. F. Ecoturismo: uma Proposta que Necessita de Planejamento. Eixo Temático: Inovação e Sustentabilidade. Fórum Internacional Ecoinovar Santa Maria/RS - 26 a 28 de Agosto de 2015. Disponível em: http://ecoinovar.com.br/cd2015/arquivos/resumos/ECO849.pdf.

KUNDLATSCH, C. A.; VIEIRA. S. R.; MOREIRA. J. C. Painéis interpretativos: ferramentas de educação ambiental no turismo em áreas naturais. Cadernos de Estudos em Pesquisa de Turismo. Curitiba, v.5, n 7, p. 34-50, jul/dez. 2016. 
MMA - Grupo de Trabalho Interministerial. Diretrizes para uma Política Nacional de Ecoturismo. Brasília. 1994. Disponível em: https://www.mma.gov.br/estruturas/sedr proecotur/ publicacao/140 publicacao20082009043710.pdf. Acesso em $28 / 11 / 2016$ as $22 \mathrm{~h} 28 \mathrm{~min}$.

OLIVEIRA, C. C. V.; CENDÓN, B. V.; CIRINO, S. D. Aspectos estruturais considerados nos estudos de qualidade dos periódicos científicos. Pesquisa Brasileira em Ciência da Informação e Biblioteconomia. v. 12, n. 1, p. 094-106. João Pessoa, 2017.

PAULA, G. DA C.; ALBACH, V. DE M. A Produção Acadêmica Sobre a Educação Ambiental e Turismo: Um Estudo Bibliométrico. IX Fórum Internacional de Turismo do Iguassu 17 a 19 de junho de 2015 Foz do Iguaçu - Paraná - Brasil. Disponível em: http://bit.ly/paula-albach

SALES, D. S. Critérios de avaliação da produção científica em ciências sociais aplicadas: inquerindo as bases de dados. Universidade de São Paulo, escola de comunicações e artes. São Paulo 2013. Disponível em: http://doi.org/10.11606/D.27.2013.tde-26052015-122438

SARCADO, M. S.; HAYASHI, M. C. P. I. A pesquisa em educação física na interface com a educação na região centro-oeste do brasil: uma análise epistemológica e bibliométrica. In: Colóquio de Epistemologia da Educação Física, 5., 2010, MaceióAL. Anais... Maceió-AL, 2010

SCIELO - Scientific Electronic Library Online. Critérios, política e procedimentos para a admissão e a permanência de periódicos científicos na Coleção SciELO Brasil. São Paulo: SciELO, 2014

TERENCE, A. C. F.; ESCRIVÃO FILHO, E. Abordagem quantitativa, qualitativa e a utilização da pesquisa-ação nos estudos organizacionais, ENEGEP, 26. 2006, Fortaleza, CE, 2006.

VALERIO, P. M. Espelho da ciência: avaliação do programa setorial de publicações em ciência e tecnologia da Finep Rio de Janeiro: Finep, 1994.

VASCONCELOS, J. M. O. Interpretação ambiental. In: MITRUAD, S. (org.). Manual de ecoturismo de base comunitária: ferramentas para um planejamento responsável. Brasília: WWF Brasil. p. 470, 2003. 


\section{Dados dos autores}

Brenda Oliveira Nascimento

Licenciada em Educação Física pela Universidade Federal de Sergipe. Bolsista CDPPEL - Rede CEDES.

brendaedf@gmail.com

\section{Roberto Gondim Pires}

Graduação em Educação Física pela UFBA (1994). Especialista em Metodologia do ensino da Educação Fìsica pela UESB. Mestrado em Educação: História, Política, Sociedade pela PUC-SP (2001). Doutorado em Educação:Gestão e Política da Educação pela UFBA (2007).

Gondim.roberto@gmail.com

Robelius De-Bortoli

Licenciado em Educação Física - UFRGS. Especialista em Futebol Administração e Marketing - UGF. Doutor em Ciência de la Actividad Física - UNILEON. Pos-Doutorado pela Universidade da Costa Rica.

robelius@yahoo.com.br

Received - Recebido: 2017-09-05

Accepted - Aceitado: 2018-12-14

\section{Agradecimentos}

Centro de Desenvolvimento de Pesquisas em Políticas de Esporte e Lazer de Sergipe - CDPPEL

\section{(cc) BY}

This work is licensed under a Creative Commons Attribution 4.0

United States License.

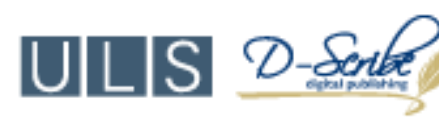

This journal is published by the University Library System of the University of Pittsburgh as part of its D-Scribe Digital Publishing Program and is cosponsored by the University of Pittsburgh Press. 